\title{
Mutually coordinated visualization of product and supply chain metadata for sustainable design
}

\author{
William Z. Bernstein;, Devarajan Ramanujan \\ School of Mechanical Engineering \\ Purdue University, West Lafayette, Indiana 47906 \\ Devadatta M. Kulkarni, Jeffrey Tew \\ Cincinnati Innovation Lab \\ Tata Consultancy Services, Milford, OH 45150 \\ Niklas Elmqvist \\ College of Information Studies \\ University of Maryland, College Park, MD 20742
}

\author{
Fu Zhao ${ }^{1,2}$, Karthik Ramani ${ }^{1,3}$ \\ ${ }^{1}$ School of Mechanical Engineering \\ ${ }^{2}$ Division of Environmental and Ecological Engineering \\ ${ }^{3}$ School of Electrical and Computer Engineering (by courtesy) \\ Purdue University, West Lafayette, Indiana 47906
}

\begin{abstract}
In this paper, we present a novel visualization framework for product and supply chain metadata in the context of redesign-related decision scenarios. Our framework is based on the idea of overlaying product-related metadata onto interactive graph representations of a supply chain and its associated product architecture. By coupling environmental data with graph-based visualizations of product architecture, our framework provides a novel decision platform for expert designers. Here, the user can balance the advantages of a redesign opportunity and manage the associated risk on the product and supply chain. For demonstration, we present ViSER, an interactive visualization tool that provides an interface consisting of different mutually coordinated views providing multiple perspectives on a particular supply chain presentation. To explore the utility of ViSER, we conduct a domain expert exploration using a case study of peripheral computer equipment. Results indicate that ViSER enables new affordances within the decision making process for supply chain redesign.
\end{abstract}

Keywords: sustainable design, visual analytics, LCA interpretation, supply chain design

\footnotetext{
*Address all correspondence to this author. wbernste @ purdue.edu
}

\section{Introduction}

With future environmental regulations imminent in the United States, companies are faced with the need to improve existing product systems for environmental performance, something which often presents difficult decision scenarios [1]. The complexity of these scenarios is compounded once the supply chain is taken into consideration. Even after conducting a full-fledged life cycle assessment (LCA), it is still difficult to identify feasible redesign opportunities, including balancing cost and operational performance with environmental performance. Furthermore, interpreting a product system's environmental profile has been a significant challenge since the release of the ISO 14000 series [2], which within provides general guidelines for conducting an LCA. To date, many impact assessment methods, such as the Environmental Protection Agency's (EPA) Tool for the Reduction and Assessment of Chemical and other environmental Impacts (TRACI), express environmental impact through various impact categories, essentially presenting a multi-criteria decision problem for the practitioner. Even so, LCA has become the most accepted method for assessing the environmental impact of products, processes and systems. However, the ISO standards do not directly provide direction to the practitioner for conducting such analysis. As a result, discretion is often left to the practitioner.

Current LCA platforms and methodologies can inform redesign practices, but the lack of intuitive data representa- 


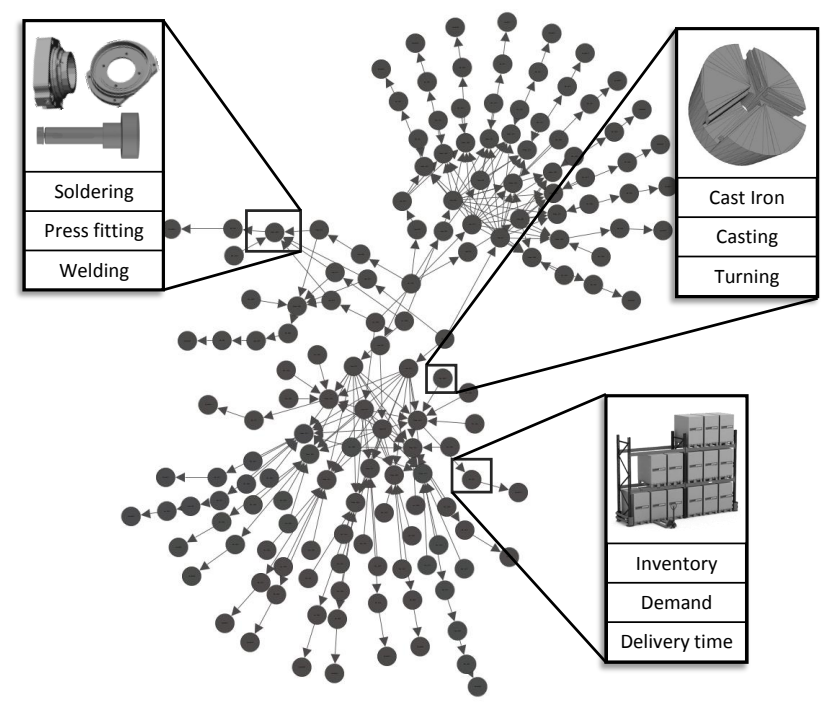

Fig. 1. Graph-based visualization of a real-world supply chain of computer peripheral equipment [4]. Each node carries product metadata depending on the supply chain echelon type, such as an assembly stage, part manufacturing, and warehousing.

tions create decision-related barriers. In order to overcome these challenges, we use guiding principles from the fields of visual analytics and information visualization (InfoVis), to develop an interactive visual analytics framework that supports eco-conscious redesign for supply chains. Our platform focuses on interpreting product-level attributes, such as LCA impacts, based on the structure of a supply chain and its product's architecture. To this end, our framework offers dynamic visualizations of LCA data from multiple perspectives, allowing the user to discover anomalies and understand redesign implications. This allows decision makers to better understand the impact of redesigning a component and how the proposed change propagates through the rest of the product system. Our aim is to lessen barriers associated with the interpretation stage of LCA, enabling redesign-related decision making for stakeholders.

In order to demonstrate our framework, we present a prototype software tool, coined ViSER, Visualizing Supply chains for Eco-conscious Redesign. ViSER is an interactive visualization tool that provides an interface consisting of multiple mutually coordinated views providing different perspectives on a particular supply chain presentation. In this context, mutual coordination refers to maintaining a link of each user interaction with respect to each visualization output, including the resulting views as well as the back-end data. Throughout the development of ViSER, we focused on understanding how users interact with design data during the platform's use. There exist trade-offs between software features and cognitive load [3]. The bridge between the two is significant in producing design tools that engineers with limited training in an application domain, such as environmental sustainability, can properly use.

The contributions of this work include (1) a data representation method that enables mutually coordinated visualizations for adjacency matrices of a product system and its supply chain, (2) a mapping scheme for visual variables to product and supply chain metadata, and (3) a visual analytics-based prototype tool for interactive exploration of supply chain metadata.

This paper presents a decision-making framework for eco-conscious supply chain redesign. Therein, we embed a methodology that enables users to balance multiple criteria associated with a product system, including its supply chain, for creating effective redesign scenarios. First, the motivation and related work surrounding relevant areas of research is presented. Then, we present the core methodology for obtaining relevant product metadata, appropriately mapping this data to visual variables, and visually presenting data representations to the decision maker. Finally, we present the implementation of our framework through the ViSER tool. In order to validate our method, we conducted a task-oriented user study consisting of expert participants from industry.

\section{Motivation}

Figure 1 provides a visual representation of a real-world supply chain network of computer peripheral equipment [4]. Herein, all supply chain stages with their associated metadata are represented. Throughout this paper, we refer to 'metadata', or data about data, which describes variables and attributes associated with component and supply stage data types. In the context of product redesign, understanding the effects of changing a particular component with respect to the rest of its product system is quite difficult, as there exist both indirect and direct relationships with other network entities. When considering metadata associated with each node, such as material and manufacturing processes, the complexity for such redesign scenarios is compounded since these attributes could depend on spatial and temporal constraints. Hence, there is a need for the development of more effective and transparent techniques to represent these graphs in the context of redesign planning, enhancing the visibility of the data directly to the decision maker.

There have been efforts within current computer-aideddesign (CAD) platforms that offer retroactive features to help designers explore "what-if" scenarios for downstream design modifications ${ }^{1}$. In general, these tools simplify the redesign process by only allowing point substitutions, such as changing the material type, a single manufacturing process or the distribution mode of individual parts. Furthermore, there is currently no widely accepted method for determining how such changes influence the product system and its supply chain. As a result, it is necessary to provide a basis for designers to understand how a change in either domain affects the system's overall environmental performance.

With respect to LCA, developing a weighting scheme for impact damage categories to aid in decision making is

\footnotetext{
${ }^{1}$ e.g. SOLIDWORKS Sustainability Xpress, Granta's Eco Audit ${ }^{\mathrm{TM}}$
} 
quite difficult. There have been considerable efforts to categorize damage categories into high level scores with weighting techniques. The issue is that these single scores are based on estimations and assumptions that provide decision makers limited flexibility depending on their own value system. Software platforms that support LCA, such as SimaPro and $\mathrm{GaBi}$, offer their own visualizations for reporting results, including single score metrics. NIST's BEES software, for example, offers dynamic, user-defined weighting schemes for impact categories. However, there is a trade-off between providing freedom to the practitioner and grounding the results in an acceptable theory [5].

Beyond the interpretation of LCA results, we aim to provide a novel sustainable redesign methodology for complex product systems by visually overlaying metadata onto graphical representations. Here, we use environmental analysis as a target, since it inherently carries multi-variate data. However, our approach can be extended to any decision scenario related to supply chains.

\section{Related Work}

Integrating product architecture with supply chain design is a complex problem. There has been considerable work in modeling products and their supply chains to further understand these systems. As an example, modeling supply chains as networks has led to the development of various criticality and complexity metrics to better understand supply network configuration [6]. In scenarios where large complex and heterogeneous datasets exist, such as product systems, visual analytics has proven to alleviate user cognitive load and expedite useful discovery by projecting emergent relationships between entities [7]. However, the application of these guiding visualization principles to engineering systems remains in a nascent stage. The following sections review relevant literature related to understanding and communicating the underlying structure of supply chains. For this, we look at prevalent engineering metrics and visualization techniques associated with product-related data.

\subsection{Modeling supply chains}

There has been considerable work targeted at modeling supply chains at various abstraction levels in order to develop and validate metrics, such as complexity measures. As an example, dependency matrices have been used to develop complexity metrics within project management [8], supply chains [9] and manufacturing [10]. Similar methods have been used to understand supplier network relationships through weighted adjacency matrices [11]. These efforts are from an operations research perspective and aim to measure static network attributes, such as modularity. Additionally, recent techniques have been developed to connect product complexity and supply chain impacts. Inman et al. studied the probability of disrupting a supply chain by relating the likelihood of an individual part missing within a specific supplier [12]. Wagner and Neshat established a method for assessing the vulnerability of a supply chain us- ing a graph-theoretic approach with adjacency matrices [13]. Recent work has focused on proper visualization techniques for matrices, networks, or a combination of both $[14,15]$, suggesting that unsupervised frameworks alone are not the best option.

\subsection{Visualizing supply chains}

Supply chains have been studied as an application area within the information visualization (InfoVis) community. For example, Minegishi and Theiel represented supply chain interactions, such as cost trade-offs in production, within a causal loop diagram [16]. Recently, there has been a push to include geo-spatial data, often through geographic information systems (GIS), to visualize supply chains across multiple dimensions [17]. Hu et al. developed a framework for visually representing geographical attributes of a supply chain using a case study from the transport container industry [18]. In another example, Kassem et al. developed a visualization scheme for mapping relevant information to the progress of constructing a building, including the supply chain [19].

Other work focuses on developing platforms to aid decision making for supply chains. TISCSoft is a decision support tool to help optimize transportation infrastructure within a supply chain. Demand is shown by node sizing superimposed onto a map with distribution locations allowing the user to internalize multiple data entities at once [20]. Lin et al. described IBM's efforts in representing traditional inventory management information in dynamic interfaces [21]. Others have used similar ideas to improve the environmental sustainability of supply chains, e.g. towards innovation potential [22] and modeling carbon footprints [23]. MIT's Media Labs developed Sourcemap, a material-focused supply chain tool that allows the user to understand eco-costs per supplier [24]. Although Sourcemap provides an interactive visualization environment, the connection between projecting design changes to supply networks is missing. Sourcemap does not provide any detailed information pertaining to the product itself, making it a less viable candidate for a suitable decision software platform for engineers.

\subsection{Aligning product architecture with its supply chain}

Aiming to contextualize the aforementioned efforts within design practices, previous studies have focused on establishing methods that inform supply chain configuration based on product architecture. Hu et al. proposed a method to meet customer requirements related to product variety with a complexity threshold of its resultant supply chain [25]. Nepal et al. presented a weighted goal programming model to optimize supply chain structure based on product architecture [26]. Khan et al. presented a case study within the fashion design industry that aligns product design strategy with supply chain structure [27]. Ülkü et al. investigated how product architecture, and modularity specifically, affect the performance of the supply chain [28]. Others have focused on the inverse of this research problem, through understanding how changes to the design of a product impact downstream activities. 


\subsection{Visually mapping engineering-related data}

Although our work does not directly focus on change propagation within engineering systems, we incorporate such considerations into our framework. Existing work presents various methods for assessing the impacts on design changes within complex systems [29]. However, creating dynamic visualization interfaces that represent such metrics and, in turn, keep the human user in the sensemaking loop [30] is still in its infancy. Keller et al. [29] extend their work in change propagation with a few prototype visualization interfaces for use in design [31]. Giffin et al. investigated the use of the design structure matrix to assess change propagation through a complex engineering system [32]. Here, a visualization of an adjacency matrix of all proposed engineering changes was used to assess which specific entities are at particular risk. Other studies show a form of change propagation by visually representing hypothetical situations. Goodwin et al. developed a visualization tool for users to explore "what if" scenarios regarding their daily energy consumption in their homes [33]. Another study presented diagrams for illustrating relationships between entities within a development project to enhance design teams' activities [34]. Additionally, NIST released a visualization framework for querying standards associated with sustainable manufacturing, which could be extended to complex supply chains [35].

Contributions from the InfoVis and engineering design communities seem to be separated in "silos". Alternatively, efforts from the InfoVis community seem to be too focused on geo-spatial layout and do not display implications of supply chain changes to product architecture and vice versa. Our goal is to contribute to these efforts by incorporating guiding InfoVis principles within engineering design and, in particular, a sustainability context. We envision that our work can push towards a set of unified visual representations to aid in concurrent understanding of both product and supply chain structure [36].

\section{Methodology}

Our approach, illustrated in Fig. 2, consists of a data handling pipeline with the goal of projecting manipulable and easy-to-understand data schemes to the user. Given product and supply chain configurations in the form of adjacency matrices, we propose a framework for the interpretation of associated environmental data. Here, we present two separate modules, including a user-directed visualization interface and a back-end data representation engine. This section will detail data flow and operations through the exploration process offered by our framework. All process steps discussed are directed to developing a platform that enables interactive user sensemaking for the eco-conscious design of supply chains and their product systems.

\subsection{Procuring data from existing databases}

First, we assume that data is housed within a product database management (PDM) system carrying meta-

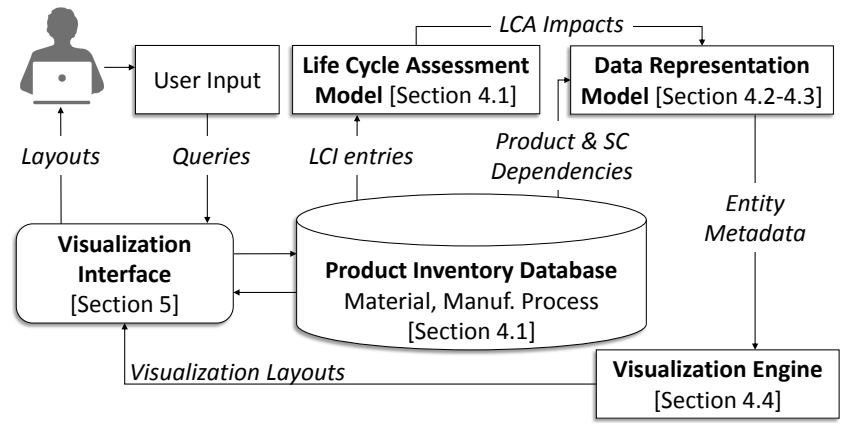

Fig. 2. The general pipeline of the data handling processes associated with our proposed visualization framework. Each module, shown in bold, it explained within the provided section callout.

data associated with products and their respective supply chains. This information includes a complete Bill of Materials (BOM), comprising of material type and all primary, secondary and auxiliary manufacturing processes. Ideally, this inventory database would encompass relationships associated with entities of the product system and supply chain. For the product system, we can construct its graphical representation, through an adjacency matrix, representing structural relationships. With respect to the supply chain, its graphical representation is described through product flow from procurement to its eventual distribution.

Based on data from the PDM, life cycle inventory (LCI) inputs can be extracted for environmental assessment. In the future, we envision that existing LCA tools, such as $\mathrm{GaBi}$ and SimaPro, to interface with PDM systems for integrated analysis, potentially including real-time, parametric LCA. Within our framework, we extract LCI inputs based on the appropriate level of data granularity and conduct an LCA in order to procure data associated with significant damage categories. Our methodology allows for alternative assessment techniques, such as an economic input-output LCA (EIO$\mathrm{LCA}^{2}$ ), particularly for situations where data is incomplete, or when the entire BOM is not available.

\subsection{Presenting product entities as objects}

A primary contribution of this paper is the idea of overlaying data attributes onto graphical representations of product entities. This fundamental step allows the user to directly interact with attributes. To this end, we develop a Data Representation Model (DRM) that organizes all product data, both directly extracted from the PDM as well as computed metrics, such as midpoint environmental indicators from LCA. Figure 3 provides a UML-based diagram of the data architecture presented throughout this paper. Here, we present both supply chain stages and product entities (i.e. components or sub-assemblies) as objects, which are aggregated into the supply chain and product system networks, respectively. All metadata procured from the PDM are handled as data attributes per object, such as an idNumber and manufProcess. It should be noted that additional metadata can be

\footnotetext{
${ }^{2}$ http://www.eiolca.net/
} 


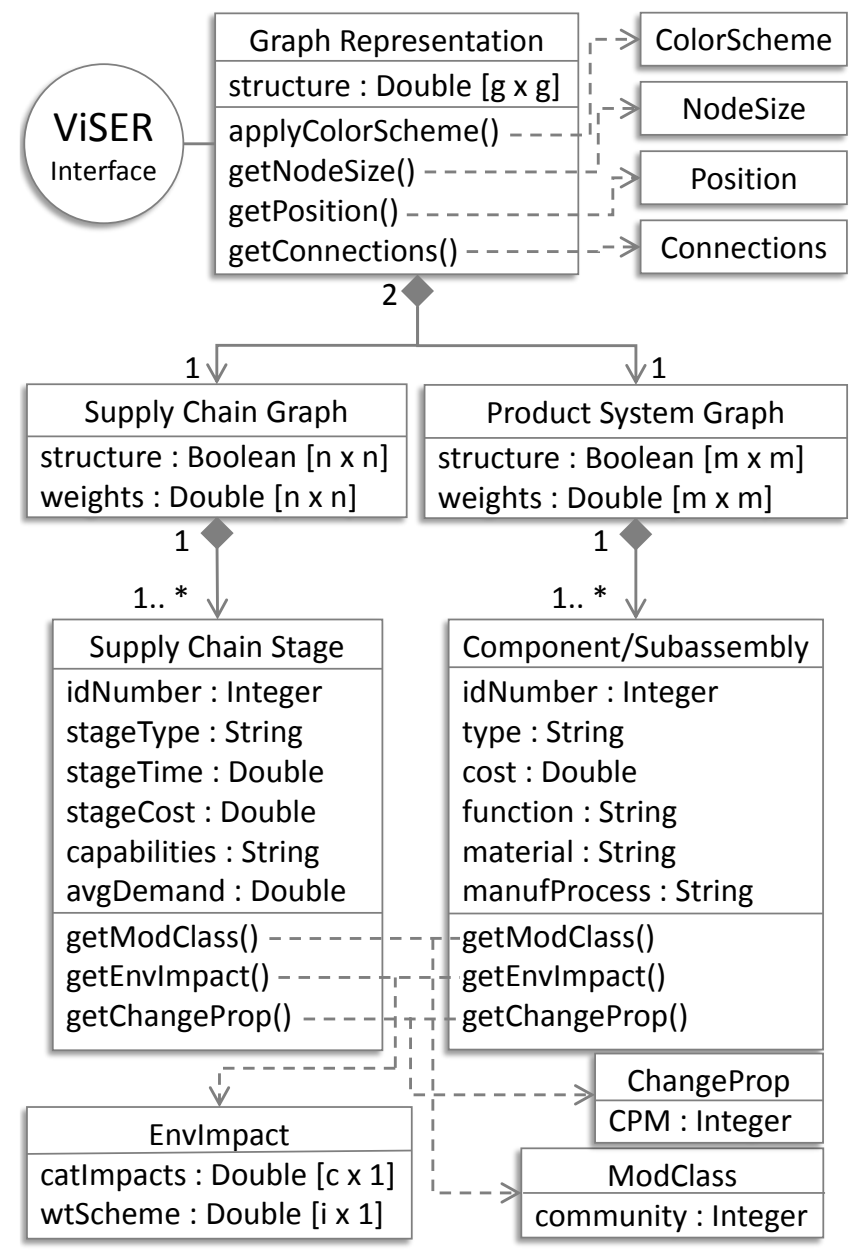

Fig. 3. UML-based depiction of data representation in ViSER. Here, we show metadata that were incorporated into the prototype.

incorporated into this framework based on the context and domain. Computed metrics are coded as separate functions, such as EnvImpact and ModClass, in which all input parameters are available from the object level or its composition, or its network structure. Our framework's data architecture enables the assignment of visual variables to specific data attributes, such as node size and color. This is explained in more detail in Section 4.4.

Within the DRM, we implement several data related metrics to augment the sensemaking process: (1) a modularity index that defines sub-families within the supply chain graph, (2) a change propagation metric that represents connectivity for both the product and supply chain representations and (3) contextual LCA data. For the modularity metric, we recognize that there are various available assessment actively used throughout practice and research. In our model, we implement the Louvain method [37] since it does not require an initial estimate of the number of clusters and offers community detection with a non-weighted adjacency matrix.

\subsection{Assessing connectivity for graphical representations}

Here, we discuss techniques to estimate the propagated effect associated with a redesign change throughout the supply chain. Assessing the risk associated with a product's architecture after an engineering change has been well studied as discussed in Section 3.4 [32]. However, to properly conduct a change propagation analysis, considerable knowledge of the product is required. As discussed, within a PDM system, the material and manufacturing attributes related to a product can be procured. Likewise, physical relationships between components are captured via an adjacency matrix.

Since the supply chain and product system can be represented as graphs, we can adopt accepted graph-theoretic techniques to assess the relative connectivity of vertices with respect to the entire graph. It is our assumption that redesigning more heavily connected supply chain entities would exhibit a more significant propagated effect. As a result, our metric is based on node degree. Given an adjacency matrix of graph, $G$, we can compute the total number of paths of length up to $k$ by Eq. 1 [38].

$$
\operatorname{adj}\left(G^{k}\right)=\sum_{i=1}^{k}[\operatorname{adj}(G)]^{i}
$$

The metric presented in Eq. 1 represents neighborhood closeness where directionality is not considered.

In the case of the supply chain graph, where edges have a specific direction, it may be more appropriate to account for directionality in order to avoid counting infeasible paths. As an example, PageRank offers a centrality assessment most applicable for directed graphs [39]. This is simply one assessment technique to judge the connectivity (or centrality) of vertices in a directed graph. For large networks, as an example, these metrics can be estimated based on specific graph attributes, such as material flow, supplier risk and cost.

\subsection{Assigning visual variables to metadata}

In order to map visual variables to appropriate graphical data, we recall Jacques Bertin's original seven basic visual units: position, size, shape, value, color, orientation, and texture [40]. The idea is that adjusting the values of these visual variable types can inform the presentation of data. Choosing which visual variable would be most appropriate for representing various aspects of the information is critical. In order to guide selection, Bertin offered a list of visual variable characteristics: selective, associative, quantitative, order, and length. The first four characteristics classify visual variables into effective means of grouping data appropriately. For example, a selective variable should allow the decision maker to quickly identify a modified entity from all other entities. Bertin also provides an estimated resolution, or length, for each characteristic. As far as mapping the efficacy of each visual variable to each characteristic, Fig. 4 details which variable types are appropriate for specific characteristic schema [41]. Figure 4 also summarizes each characteristic per variable type with an example. 


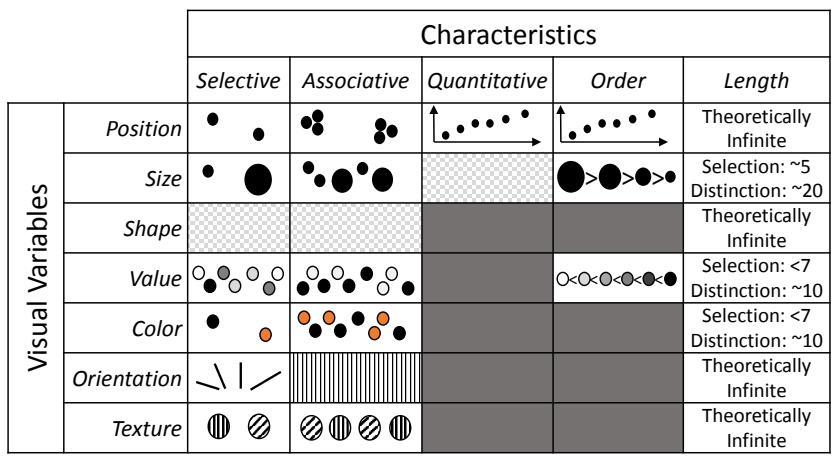

Fig. 4. Visual variables with characteristics based off of Jacques Bertin's basic visual units, adapted from Carpendale [41].

In the context of networks, we can leverage these guiding principles. Firstly, position plays a significant role in a supply chain network from two perspectives: the geo-spatial location of a particular echelon and its grouping with respect to other stages in the product system. The latter might help present specific network modules, or sub-assemblies. For quick surveying of metadata on supply chains, size can be used since humans can perceive differences in about 20 different values [41]. For observation across all entities in the network, a chart representation of criteria values can be presented to utilize position as its visual variable, which carries a theoretically infinite length for distinction. For grouping or qualitative assessment, color is appropriate and hence is relevant for describing supply chain echelon types, such as differentiating a product procurement process from a warehousing center. These established principles for graphical presentation guided our own selection for specific visual variables as representations for supply chain information.

Figure 5 provides an example of the mapping modes of visual variables to graphical representations as well as their limitations. If we assume the network in Fig. 5 to represent a simple product, wherein two sub-assemblies, i.e. [2-3-58] and [1-4-6], are connected via a common component, 7. Here, node position is representative of community membership and can be quickly conveyed to the user. The node size is directly proportional to a hypothetical metric and the color hue represents node type. It can be observed through the graph layout that Node 1 carries with it the highest value with respect to the criteria. However, differentiating Node

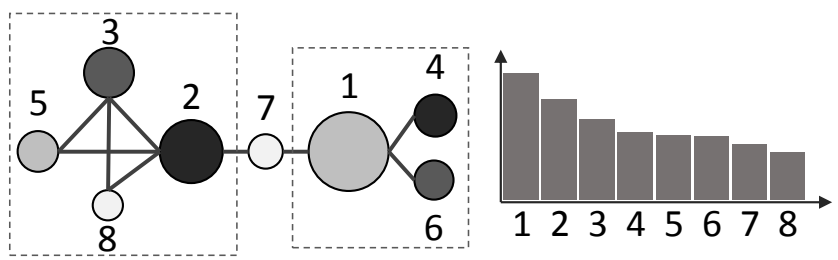

Fig. 5. Representation of mapping visual variables to graphical information. Position informs modularity, or community membership. Size is proportional to criteria of interest. Value of shading informs node type. The barchart corresponds directly to size of each node.
5 from Node 6, for example, is quite difficult. This demonstrates the significance of the perceived length of a visual variable. Though, size is theoretically infinite, it is practically limited [41]. To overcome this challenge, we provide an additional representation of the same data in barchart form.

In the next section, we introduce a prototype tool built in accordance with these guiding visual principles to ease the interpretation of environmental and performance metadata for supply chains.

\section{Implementation: the ViSER tool}

Here, we present ViSER, a prototype design tool implementing our framework. ViSER is an interactive visualization tool that provides a panel consisting of multiple mutually coordinated views providing multiple perspectives on a particular supply chain scenario. Based on the discussed challenges for designing environmentally efficient product systems we provide four requirements for our prototype tool:

R1: Augmenting the exploration process towards a potential redesign scenario.

R2: Mutually coordinating the selection of an entity in one graph with respect to the other.

R3: Aiding trade-offs between environmental and traditional performance metrics.

R4: Defining user-enabled eco-impact weighting schemes for results interpretation.

Throughout the presentation of ViSER, we refer back to these requirements for the purpose of clarity.

\subsection{Implementation notes}

This research prototype was built using Processing $2.1^{3}$. In our approach, graphs are used to visualize relationships between supply chains and its subsequent product graph. It is important to allow user interaction within the graphs to handle large complex supply chains. Here, we propose using LCA data as node attribute data, since the nature of the data presents some interesting trade-offs due to its heterogeneity. However, this design tool can be used to visualize any data associated with supply chain entities.

\subsection{Visual features for supply chain entities}

To meet R1, ViSER allows for dynamic, quick surveying of node attributes in a user-centric manner. In general, the main cognitive anchor of the tool lies within the visual attributes of graphical nodes, including size, color and position. Each feature and its intent is explained below.

- Color: We use color to identify node type within the graphs. The default coloring scheme is based on supply chain stage type, e.g. component, assembly, and retail. Additional color options are available via a checkbox, including a coloring scheme based on a clustering algorithm described by Blondel et al. [37]. ViSER offers additional coloring options based on specific stage types.

\footnotetext{
${ }^{3}$ http://processing.org
} 


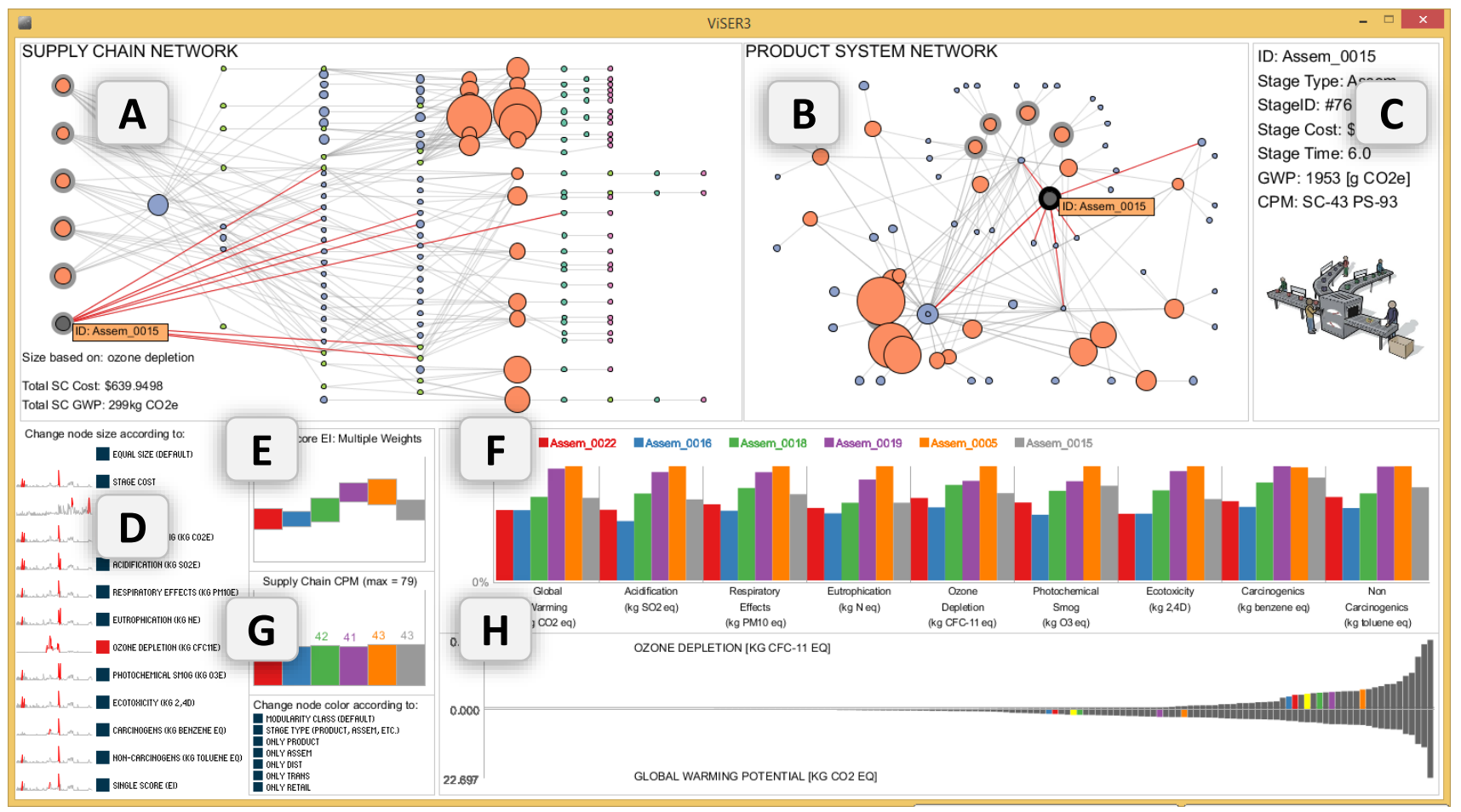

Fig. 6. Screen capture of the ViSER tool. Each feature of the ViSER platform is described as follows: (A) a directed graph that represents the supply chain network as the edges connected to the selected nodes are shown in red, (B) a representation of the product system graph oriented by a combination of force directed physics and user manipulation, (C) a panel with detailed information about the most recently selected item, (D) sparklines for each criterion with outliers shown in red, (E) a boxplot that provides a range of single score impacts per selection, (F) a comparison chart of impact categories for selected nodes, $(G)$ change propagation results for selected nodes, and $(H)$ the profile of selected node attributes, ecotoxicity. A demonstration video can be seen here: http://goo.gl/mTqbby.

- Size: Node sizing is directly proportional to the normalized value of a user-selected criterion (Fig. 6D). Node sizing in ViSER aids the user with respect to initial anomaly detection within the graphs.

- Position: We allow for dynamic node placement, where users can toggle a spring-force network layout engine ${ }^{4}$. Alternatively, the user can select a tree layout for the supply chain, which features a representation for the parent-child relationships of supply chain stages.

Aside from displaying nodes according to specific attributes, all raw data associated with a node can be accessed via the detail viewer, which is populated after selecting a particular node, as seen in Fig. 6C.

\subsection{Change propagation metric}

Within the tool, we also express an indicator for the change propagation of a selected redesign activity. For demonstration, we employ the change propagation metric $(C P M)$, based off of Eq. 1 described earlier, where $k=2$. As shown in Algo. 1, the CPM is calculated by summing each row except for the corresponding diagonal value of $A$. The diagonal elements of $A$ are omitted for the calculation because the $C P M$ should represent the total number of connections between the $i^{\text {th }}$ supply chain element and the other

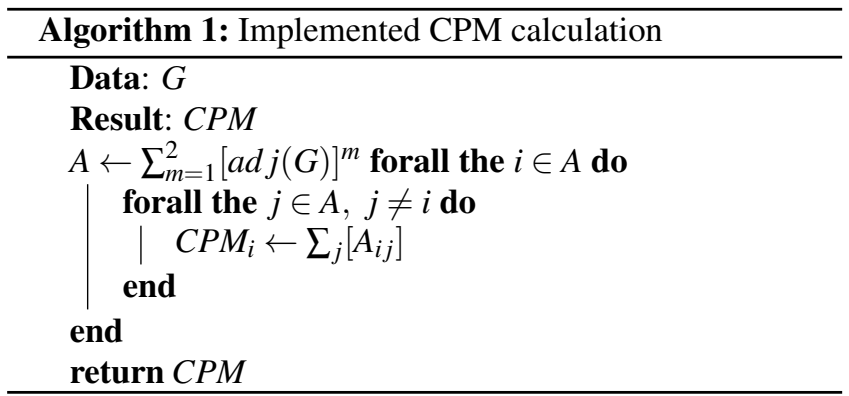

$j$ elements, excluding its relationship with itself. The algorithm requires an adjacency matrix of a graph, $G$, and outputs a column vector containing all $C P M$ values.

Additionally, in order to visualize connectivity of a particular node, all neighboring nodes of a selected node are highlighted in red, as seen in Fig. 6A-B. This allows users to survey nodes of interest and quickly gain insight into each network's structure. For the supply chain graph, when coupled with the tree layout visualization, the highlighting provides a macro-level perspective on its overall structure.

\subsection{Visually comparing entity selections}

Since one of the main goals of our platform is to compare different redesign alternatives, the ViSER tool offers 


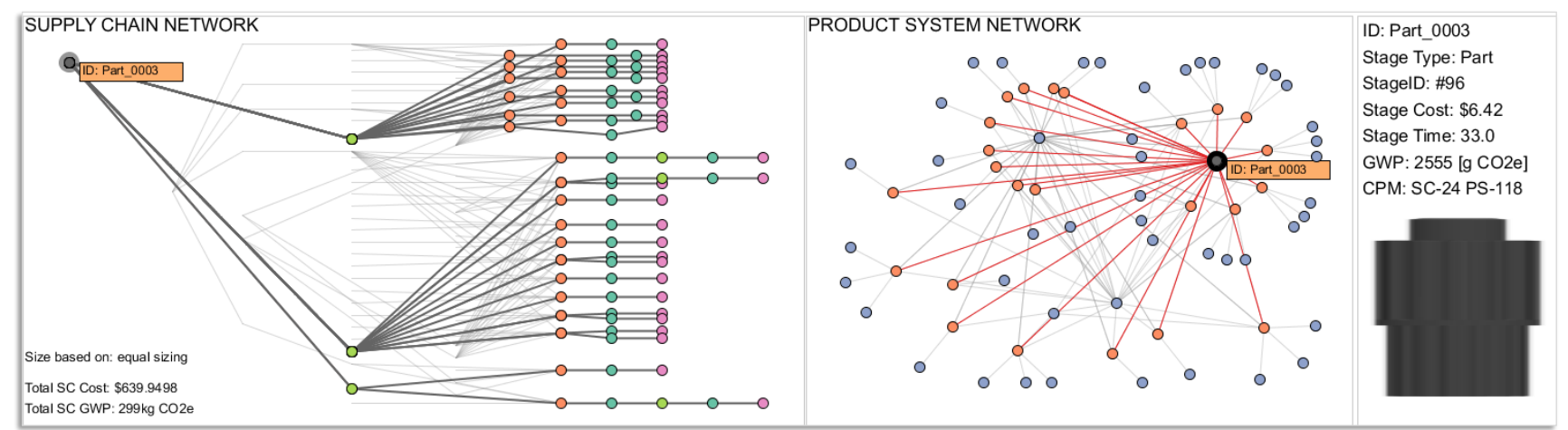

Fig. 7. Here, one can see the value chain viewer. If users wish to investigate the value chain of particular product or sub-assembly, they right click a node in the product system graph and its value chain is emphasized in the supply chain graph.

multiple visualizations for node comparison. In its current form, the prototype tool allows for comparing single node selections. Future iterations of the ViSER tool will allow multiple sets of nodes and path selections. Each visual representation illustrated in Fig. 6 is reviewed below.

- Sparklines: Anomaly detection is conducted by surveying the sparklines for each criterion, seen in Fig. 6D [42]. In the proposed case study, values that exceed five times the average of all nodes are highlighted in red.

- Profile Barchart: The profile barchart visually represents selected node values for ease of surveying. When two criteria are selected, the profile barchart splits into two adjacent charts, each representing one of the selected criteria. When hovering over a node, the appropriate bar within the chart is highlighted.

- Clustered Barchart: When two or more nodes are selected, a normalized barchart for multiple criteria is shown, as illustrated in Fig. 6F. This allows for direct comparison of multiple nodes across different criteria. Values here can be either expressed as a fraction of the maximum value in each category or only among the selected nodes.

- CPM Barchart: We compute the CPM based on each graph, both the supply chain tree and product system network, seen in Fig. 6G.

- Single Score Barchart: Based on pilot studies, we also included a barchart that shows a minimum and maximum value of the single score environmental impact. We compute differently weighted single scores based on four commonly applied TRACI weighting schemes [5].

- Value chain viewer: Figure 7 illustrates the value chain viewer implemented in ViSER. After right clicking an already selected node, the value chain for which that node belongs is highlighted within the supply chain. This allows users to quickly filter out entities within the supply chain.

Furthermore, as the user hovers over a node, the ID label associated with that entity is shown on both graphs to provide an understanding of its role in both contexts. Additionally, the edges directly connected to that node are shown in red to give a representative idea of the connectivity of that node. For example, a component that is shared across multiple sub-assemblies could be associated with several distribution pathways. The coordination of ViSER's features provide multiple data exploration affordances, fulfilling R2 and R3.

\section{Case study}

In order to demonstrate the usefulness of ViSER, we conducted a case study on a supply chain for computer peripherals. Here, we describe the dataset used with necessary pre-processing work and limitations related to the data.

\subsection{Dataset details}

Here, we implement a supply chain example that is representative of the peripheral computer equipment industry released openly at INFORMS 2008 [4]. The original dataset provides the connectivity of each node in the supply chain, along with the cost and time associated with each supply chain stage. The original dataset also provides information regarding the average demand at each retail stage. Within each supply chain model, there are five entity types:

- Dist_: a stage that stores and distributes an item.

- Manuf_: a stage that manufactures or assembles an item.

- Part_: a stage that procures an item.

- Retail_: a stage that acts as a demand origination point.

- Trans_: a stage that transports an item between stages.

For demonstration, this specific supply chain model was chosen since it consists of enough entities to provide interesting trade-off scenarios and, at the same time, does not cause significant graph layout problems. However, this prototype can be used to analyze any supply chain model that includes relationships between stages with minor pre-processing work.

\subsection{Inferring product graph and eco-impacts}

The original dataset does not contain information regarding the corresponding product system graph (or an adjacency matrix representing product structure relationships) associated with the supply chain. Hence, it was required 
to generate a synthetic product system graph that is representative of the actual adjacency matrix. The product system graph was generated by assuming that all manufacturing stages in the supply chain to be representative of subassemblies in the product system. In other words, the product graph in our implementation represents the physical relationships of all components and sub-assemblies. We argue that the resultant, synthetic graph still illustrates the utility of the tool and enables observation of user interactions.

Ideally, product information would be available in order to conduct a detailed life cycle assessment (LCA), in which each component is assessed based on its material, corresponding manufacturing processes, geometric information and transportation details. As we do not have access to this information, we conducted an EIO-LCA using the web-tool from Carnegie Melon University. Using EIO-LCA, we estimated environmental impacts associated with the cost of each stage in the supply chain. Since the impacts are calculated based on a purchasing price, many of the entity attributes related to environmental impact directly scale with their cost. A more detailed LCA could pose more complex trade-off scenarios.

\section{Expert review of the ViSER interface}

In order to validate the interactive features initially offered though ViSER, we conducted a user evaluation of the tool. The purpose of this study is to understand the applicability of the prototype by instructing participants to complete specified tasks related to metadata exploration. Since our goal was to elicit general concerns and issues from the participants rather than measure direct performance, we adopted an expert review approach. In general, expert reviews are known to be appropriate for situations where the interface or system to be evaluated required specific domain knowledge, such as product architecture and supply chains [43]. Typically, expert reviews are conducted using a small cohort of participants without an aim of strictly evaluating performance, such as time to complete a given task [44].

\subsection{Apparatus and software}

We conducted the user study on a Lenovo Y410p laptop with a 14" display. All user interactions with the software prototype were captured via a log file and an audio recording of each session was saved to capture intent. We periodically requested the participants to voice their opinions about each individual task and the interface at large.

\subsection{Participants}

A total of 6 domain experts ( 2 male, 4 female) were recruited to evaluate the applicability and usefulness of ViSER in an industry setting. All participants were employees of a leading provider of engineering consulting services and had prior experience in product and supply chain design. The cohort of participants exhibited a wide range of expertise level (1-15 years of experience) and age group (20-55 years old). We ensured that each expert had not been aware of ViSER or the overarching framework beforehand. Furthermore, experts were required to maintain confidentiality of the system and study amongst the other participants.

\subsection{Tasks}

Each expert was asked to complete 3 main tasks with the goal of evaluating various elements related to ViSER. Each user session began with a 15 -minute discussion on the various tool features led by the proctor. Afterwards, each participant was given 5 minutes for familiarizing themselves with the interface. Therein, the experts could freely explore each panel and begin to understand how the tool's features coordinate with one another. For each task, the initial orientation of the product graph was generic in order to allow for more "free form" exploration. This was essential for priming participants' general understanding and fluidity with regards to the interface. Directly after each task, the participant filled out a NASA Task Load Index (TLX) survey in order to record user perception with respect to the following categories: mental demand, physical demand, temporal demand, performance, effort, and frustration [45].

- Task 1: Identifying the most critical supply chain entities: The proctor asked participants to rank the three most heavily contributing supply chain stages with respect to environmental impact. The participants were offered direction as to how to complete the task, either by using the clustered bar chart reflecting values from all impact categories, the profile barchart of a selected criteria and a weighted single score estimation that reflects across all damage categories. For the second part of the task, the participants are asked to rank the three heaviest contributors to environmental impact of a particular stage type, such as an assembly stage.

- Task 2: Selecting a feasible redesign opportunity: The participant was asked to choose a particular supply chain entity that presents an appropriate redesign opportunity, primarily targeting eco-impact mitigation. Here, the participant was encouraged to balance environmental impact metrics against traditional ones, such as stage cost and stage time. Further, the proctor reminded the users about the CPM, which provides a node centrality measurement for both the supply chain graph and the product system graph, separately. The participants were instructed that they should assume that an entity with a high CPM is more difficult to redesign since there are propagated effects across either network.

- Task 3: Investigating impacts of various value chains: The participant was asked to investigate a value chain of one component in the product system graph. The proctor instructed to use stage time as the criteria for emphasizing product system entities. The goal here was specifically to identify a value chain that has a low CPM and significant potential for environment impact mitigation.

After Task 3, the participants were asked to fill out a survey based off of the System Usability Scale in order to assess the efficacy of the tool itself irregardless of the task [46]. 

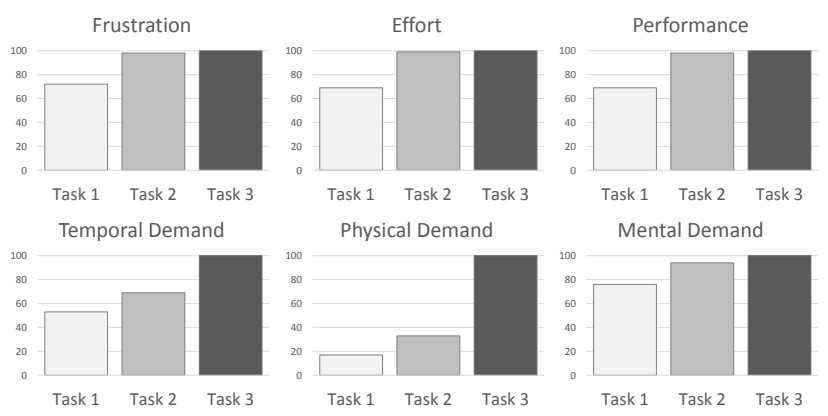

Fig. 8. Results from each NASA TLX survey given after each task. The reported task loads were pooled for each category and then normalized against the highest reported value amongst all tasks.

\subsection{Limitations of user study}

The main purpose of this study is to validate the usability of the prototype tool and evoke responses from the participants about the new interaction schemes enabled by the interface. Since (1) we are not measuring performance in terms of time required to complete a specific task, or any other quantitative metric and (2) our prototype is meant for exploration, a smaller participant population is valid [47]. Furthermore, the entire participant pool is from a particular company in industry. As a result, there might be some hidden biases in their interaction methods. Due to this small sample size, we can not make specific claims about how this tool compares to other alternative redesign assessment techniques. Even if the sample size was significantly larger, comparing our framework to alternatives is challenging because (1) we are not aware of similar existing methods for mutually coordinated visualizations of product systems and their supply chain and (2) the lack of real-world data prevents us from creating goal oriented selection tasks. In such settings, creating a quantitative comparison can lead to misleading results. Additionally, we see potential in the possible development of a synthetic benchmark that can better mimic real-world data. It is our hope that these developments will help address data availability and allow for quantitative comparisons between different methodologies present in sustainable design literature.

\subsection{Results}

Figure 8 summarizes the results of all 3 TLX surveys amongst the 6 experts. Therein, all users were pooled by summing all scores in each category. As the study progressed, participants reported a higher task load. For example, temporal demand amongst all participants was reported to nearly double from Task 1 to Task 3 . This is expected since Task 2 and Task 3 present complex decision scenarios and require the use of more features provided by ViSER. It should be noted that we asked the participants to write down specific answers to the questions posed with the tasks. When coupled with the experts' formal responses (Fig. 9), we gain more insight. Throughout Task 1, there was only 1 of 36 total responses that deviated amongst the pool of experts. Within Task 2 and Task 3, demonstrated in Fig. 9, half of

\begin{tabular}{|c|c|c|c|c|c|c|c|c|}
\hline & \multicolumn{6}{|c|}{ Task 1} & \multirow[b]{2}{*}{ Task 2} & \multirow[b]{2}{*}{ Task 3} \\
\hline & $T 1.1$ & $T 1.2$ & $T 1.3$ & $T 1.4$ & T1.5 & $T 1.6$ & & \\
\hline \multicolumn{9}{|l|}{ U1 } \\
\hline \multicolumn{9}{|l|}{ U2 } \\
\hline \multicolumn{9}{|l|}{ U3 } \\
\hline \multicolumn{9}{|l|}{ U4 } \\
\hline \multicolumn{9}{|c|}{ U5 } \\
\hline U6 & & & & & & & & \\
\hline & st & mo & & & & & $\begin{array}{l}\text { differ } \\
\text { on ans }\end{array}$ & $\begin{array}{l}\text { tes from } \\
\text { er task }\end{array}$ \\
\hline
\end{tabular}

Fig. 9. Here, we summarize the experts' responses for each task. In Task 1, each user was required to report 6 different answers. Within both Task 2 and Task 3, only one answer per participant was required.

the responses share no consensus with each other. This suggests that as the complexity of trade-offs increases, ambiguity of the decision process and task load also increase. In the context of supply chains and product systems, this further suggests that a human decision maker is critical. The incongruity of the expert responses motivates the notion of retaining the human within complex decision scenarios.

Figure 10 provides a representation of the results from the system usability scale (SUS) survey. The SUS survey aims to provide a semi-quantitative assessment of the overall ViSER architecture. The charts on the top and bottom summarize results of questions where positive responses correlate with the participant's agreement and disagreement, respectively. One user explained that if the tasks of the user study were appropriate examples of real-world problems scenarios, then the tool is designed very well. This is also reflected by the fact that all 6 participants agreed that they would frequently use the tool. The SUS survey uncovered some drawbacks of ViSER. One participant disagreed that the system was easy-to-use and reported that they were not confident while using the system. Another participant reported that the system requires support to use. However, in general, the system performed well judging by the overview of results in Fig. 10.

Since we recorded audio from each participant study and the system captured all user interactions within a log file, we can comment on the usage patterns observed. Interestingly, ViSER offers multiple modes of decision analysis. When comparing across multiple participants, the evolution of each decision space provides unique insight. We can define a decision space in ViSER as the current selection of nodes. Within Task 1, the initial query or decision space selection various amongst participants but most often (35/36) converges to a similar response. In more complex scenarios, i.e. Task 2 and Task 3, there are multiple starting points for the decision paths and unique end points, suggesting that ViSER enables free exploration. Each expert is able to provide their own domain knowledge in order to converge on a particular solution. This provides context for a collaborative use of ViSER, in which multiple stakeholders suggest opportunities spurring conversation amongst partners.

Throughout the study, participants suggested specific 

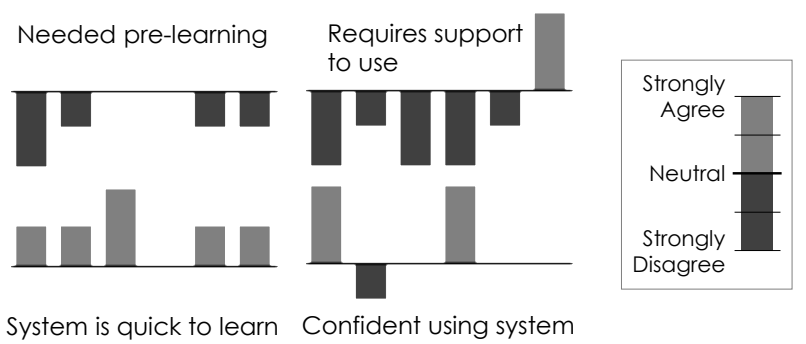

Would frequently use System is integrated

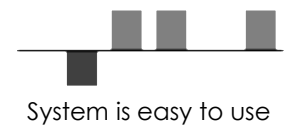

System is quick to learn Confident using system

Fig. 10. Summarized results from the System Usability Scale (SUS) survey regarding the overall functionality of the prototype software. Each bar specifies a single study participant.

improvements to the interface. These will be implemented within the next version of ViSER, as detailed below.

\subsection{Interface improvements based on user feedback}

Based on participants' suggestions from the user study, we will implement new features to the ViSER interface.

- Interactive profile barchart: Multiple users requested that the profile barchart be interactive and dynamic. For the task of finding the heaviest contributor to environmental impact of the system, one user noted that it would have been much easier to simply click the three highest bars in the sorted barchart with the corresponding nodes being selected automatically. Another participant suggested that if a user is interested in a particular node type, the profile barchart should emphasize the corresponding entries that resemble assembly stages once that color scheme is selected. Both suggestions would enhance the interactivity of the interface and seems that it would quicken sensemaking.

- Scaling node size based on CPM: In general, the overlaying of data on the graphs was most beneficial for users. As a result, some participants suggested to include both CPM metrics as options to control node sizing. A user-controlled text query in which inputs can be customized and include multiple criteria for node scaling might improve graph navigability. For example, if the user develops their own metric which carries relationships between cost, single score environmental impact and time for procurement, ViSER should allow direct overlay based on user-centered exploration.

- Panel scaling and zooming: Since this is a prototype tool, the interface is not very adaptable in terms of screen size and space. Even though it did not seem as if the size constraint affected the user studies in any way, it may be advisable to push towards a multi-window sandbox-like approach. This will potentially enable the dissemination of ViSER across a variety of visual interfaces, including tablets and large screen projectors. More user testing in how interactions scale to different display paradigms will be needed.

Improvements to the overall framework supported by ViSER are presented in the following concluding section.

\section{Conclusions and future work}

In this paper, we have presented a novel visual analytics platform for redesign-related decision making. As an implementation of these core ideas, ViSER takes advantage of existing visualization techniques to create a user-centric environment to aid multi-criteria decision making. A case study of a supply chain representative of computer peripheral equipment was used to demonstrate the usefulness. The ViSER tool enables users to identify redesign activities that minimize total environmental impact. We conducted a expert review with participants from industry to validate the applicability and usefulness of ViSER with respect to the completion of targeted tasks. Therein, participants suggested improvements to the interface based on their domain expertise.

Additionally, our framework presents a possible solution for the interpretation of life cycle assessment data of complex supply chains with multiple impact categories. The extension of user-defined weights within the ViSER interface could also provide LCA experts with a useful analytical framework. To the authors' knowledge, there is no benchmark yet accepted in the research community to handle the interpretation stage of life cycle assessment. With the advent of new open-source computer tools related to life cycle assessment, such as OpenLCA ${ }^{5}$, the development of applications aiming at facilitating decision-making beyond the presentation of data is more important than ever. To meet this need, we envision tools, similar to ViSER, to interface with inventory databases to aid environmental analyses. In the case of ViSER, we provide a visual summary of supply chain metadata to enhance the interpretation stage.

Future directions of the development of this framework will be focused on the implementation of direct operations on the supply chain and product graphs. For example, if a project stakeholder wants to delete, replace or modify a supply chain entity, the analytical model behind the visualization interface should update accordingly and present anticipated effects of that particular change. Posing such "what-if" scenarios will lead to improved, internal mental models of the decision maker. In other words, in the future, our framework can be used to introduce key learning scenarios to junior or novice engineers. The key causal relationships between graph entities can be defined by project or domain experts.

The notion of using ViSER as an exploration tool for broadening a set of possible improvements to the supply

\footnotetext{
${ }^{5}$ http://www.openlca.org/
} 
chain can be extended through a collaborative decision making process. If multiple stakeholders are interacting with the same supply chain metadata, new forms of opportunities could arise. Since our framework tracks and stores user data interactions, this collaboration could be both asynchronous and synchronous as well as co-located and geographically distributed. We envision ViSER to enable this kind of collaborative work with the core goal to keep the human in the decision-making loop. More broadly, the potential benefits of implementing visualization principles in product design scenarios provide new directions for research of large, complex, and multivariate design-related data. In addition, design and manufacturing also provide rich contexts for human-computer interaction research, especially involving people with different expertise.

\section{Acknowledgements}

This research is supported by the NSF under grants CBET 1512217, DGE 1144843, and the Tata Design Innovation Grant. The contents of this manuscript do not necessarily reflect the views and opinions of the funding agencies.

\section{References}

[1] Ramani, K., Skerlos, S., and Slocum, A., 2010. "Sustainable design?". Journal of Mechanical Design, 132(9), p. 090301.

[2] Tibor, T., and Feldman, I., 1996. ISO 14000: a guide to the new environmental management standards. Irwin Professional Pub.

[3] George Saadé, R., and Alexandre Otrakji, C., 2007. "First impressions last a lifetime: effect of interface type on disorientation and cognitive load". Computers in Human Behavior, 23(1), pp. 525-535.

[4] Willems, S. P., 2008. "Data setreal-world multiechelon supply chains used for inventory optimization". Manufacturing \& Service Operations Management, 10(1), pp. 19-23.

[5] Gloria, T. P., Lippiatt, B. C., and Cooper, J., 2007. "Life cycle impact assessment weights to support environmentally preferable purchasing in the united states". Environmental science \& technology, 41(21), pp. 75517557.

[6] Lazzarini, S. G., Chaddad, F. R., and Cook, M. L., 2001. "Integrating supply chain and network analyses: the study of netchains". Journal on Chain and Network Science, 1(1), pp. 7-22.

[7] Green, T. M., Ribarsky, W., and Fisher, B., 2008. "Visual analytics for complex concepts using a human cognition model". In Proceedings of the IEEE Symposium on Visual Analytics Science and Technology, VAST'08., IEEE, pp. 91-98.

[8] Shafiei-Monfared, S., and Jenab, K., 2012. "A novel approach for complexity measure analysis in design projects". Journal of Engineering Design, 23(3), pp. 185-194.

[9] Modrak, V., and Marton, D., 2012. "Modelling and complexity assessment of assembly supply chain systems". Procedia Engineering, 48, pp. 428-435.

[10] ElMaraghy, W., ElMaraghy, H., Tomiyama, T., and Monostori, L., 2012. "Complexity in engineering design and manufacturing". CIRP Annals-Manufacturing Technology, 61(2), pp. 793-814.

[11] Agarwal, A., Shankar, R., and Tiwari, M., 2006. "Modeling the metrics of lean, agile and leagile supply chain: An anp-based approach". European Journal of Operational Research, 173(1), pp. 211-225.

[12] Inman, R. R., and Blumenfeld, D. E., 2014. "Product complexity and supply chain design". International Journal of Production Research, 52(7), pp. 1956-1969.

[13] Wagner, S. M., and Neshat, N., 2010. "Assessing the vulnerability of supply chains using graph theory". International Journal of Production Economics, 126(1), pp. 121-129.

[14] Keller, R., Eckert, C. M., and Clarkson, P. J., 2006. "Matrices or node-link diagrams: which visual representation is better for visualising connectivity models?". Information Visualization, 5(1), pp. 62-76.

[15] Rufiange, S., McGuffin, M. J., and Fuhrman, C. P., 2012. "Treematrix: A hybrid visualization of compound graphs". In Computer Graphics Forum, Vol. 31, Wiley Online Library, pp. 89-101.

[16] Minegishi, S., and Thiel, D., 2000. "System dynamics modeling and simulation of a particular food supply chain". Simulation Practice and Theory, 8(5), pp. 321339.

[17] Greer, J., 2013. "Gis: The missing tool for supplychain design". Foresight: The International Journal of Applied Forecasting(28), pp. 44-49.

[18] Hu, Z.-H., Yang, B., Huang, Y.-F., and Meng, Y.-P., 2010. "Visualization framework for container supply chain by information acquisition and presentation technologies". Journal of Software, 5(11), pp. 1236-1242.

[19] Kassem, M., Dawood, N., Benghi, C., Siddiqui, M., and Mitchell, D., 2010. "Coordinaton and visualization of distributed schedules in the construction supply chain: A potential solution". In Proceedings of the 10th International Conference on Construction Applications of Virtual Reality, CONVR2010 Organizing Committee, pp. 77-86.

[20] Kamath, M., Srivathsan, S., Ingalls, R. G., Shen, G., and Pulat, P. S., 2011. "Tiscsoft: A decision support system for transportation infrastructure and supply chain system planning". In Proceedings of the 44th Hawaii International Conference on System Sciences (HICSS), IEEE, pp. 1-9.

[21] Lin, G., Ettl, M., Buckley, S., Bagchi, S., Yao, D. D., Naccarato, B. L., Allan, R., Kim, K., and Koenig, L., 2000. "Extended-enterprise supply-chain management at ibm personal systems group and other divisions". Interfaces, 30(1), pp. 7-25.

[22] Isaksson, R., Johansson, P., and Fischer, K., 2010. "Detecting supply chain innovation potential for sustainable development”. Journal of Business Ethics, 97(3), pp. $425-442$. 
[23] Sundarakani, B., De Souza, R., Goh, M., Wagner, S. M., and Manikandan, S., 2010. "Modeling carbon footprints across the supply chain". International Journal of Production Economics, 128(1), pp. 43-50.

[24] Bonanni, L., Hockenberry, M., Zwarg, D., Csikszentmihalyi, C., and Ishii, H., 2010. "Small business applications of sourcemap: a web tool for sustainable design and supply chain transparency". In Proceedings of the SIGCHI Conference on Human Factors in Computing Systems, ACM, pp. 937-946.

[25] Hu, S., Zhu, X., Wang, H., and Koren, Y., 2008. "Product variety and manufacturing complexity in assembly systems and supply chains". CIRP AnnalsManufacturing Technology, 57(1), pp. 45-48.

[26] Nepal, B., Monplaisir, L., and Famuyiwa, O., 2012. "Matching product architecture with supply chain design". European Journal of Operational Research, 216(2), pp. 312-325.

[27] Khan, O., Christopher, M., and Creazza, A., 2012. "Aligning product design with the supply chain: a case study”. Supply Chain Management: An International Journal, 17(3), pp. 323-336.

[28] Ülkü, S., and Schmidt, G. M., 2011. "Matching product architecture and supply chain configuration". Production and Operations Management, 20(1), pp. 16-31.

[29] Eckert, C., Clarkson, P. J., and Zanker, W., 2004. "Change and customisation in complex engineering domains". Research in Engineering Design, 15(1), pp. 121.

[30] Pirolli, P., and Card, S., 2005. "The sensemaking process and leverage points for analyst technology as identified through cognitive task analysis". In Proceedings of International Conference on Intelligence Analysis, Vol. 5, pp. 2-4.

[31] Keller, R., Flanagan, T., Eckert, C. M., and Clarkson, P. J., 2006. "Two sides of the story: visualising products and processes in engineering design". In Proceedings of the 10th International Conference on Information Visualization, IV 2006., IEEE, pp. 362-367.

[32] Giffin, M., de Weck, O., Bounova, G., Keller, R., Eckert, C., and Clarkson, P. J., 2009. "Change propagation analysis in complex technical systems". Journal of Mechanical Design, 131, p. 081001.

[33] Goodwin, S., Dykes, J., Jones, S., Dillingham, I., Dove, G., and Duffy, A., 2013. "Creative user-centered visualization design for energy analysts and modelers". IEEE Transactions on Visualization and Computer Graphics.

[34] Salustri, F. A., and Parmar, J., 2004. "Diagrammatic visualisation of early product development information”. In ASME 2004 International Design Engineering Technical Conferences and Computers and Information in Engineering Conference, American Society of Mechanical Engineers, pp. 1-10.

[35] Lechevalier, D., Narayanan, A., Reidy, S., Morris, K., and Rachuri, R., 2013. "Nist ontological visualization interface for standards: Users guide". NIST Interagency/Internal Rep.(NISTIR), 7945.

[36] Chandrasegaran, S. K., Ramani, K., Sriram, R. D., Horváth, I., Bernard, A., Harik, R. F., and Gao, W., 2013. "The evolution, challenges, and future of knowledge representation in product design systems". Computer-Aided Design, 45(2), pp. 204-228.

[37] Blondel, V. D., Guillaume, J.-L., Lambiotte, R., and Lefebvre, E., 2008. "Fast unfolding of communities in large networks". Journal of Statistical Mechanics: Theory and Experiment, 2008(10), p. P10008.

[38] Skiena, S., 1990. Implementing Discrete Mathematics: Combinatorics and Graph Theory with Mathematica. Addison-Wesley, Reading, MA.

[39] Perra, N., and Fortunato, S., 2008. "Spectral centrality measures in complex networks". Physical Review E, 78(3), p. 036107.

[40] Bertin, J., 1983. "Semiology of graphics: Diagrams, networks, maps (wj berg, trans.)". Madison, WI: The University of Wisconsin Press, Ltd.

[41] Carpendale, M., 2003. "Considering visual variables as a basis for information visualisation”. Computer Science TR\# 2001-693, 16.

[42] Tufte, E. R., and Graves-Morris, P., 2001. The Visual Display of Quantitative Information, Vol. 2. Graphics Press Cheshire, CT.

[43] Elmqvist, N., and Yi, J. S., 2013. "Patterns for visualization evaluation". Information Visualization, p. 1473871613513228.

[44] Tory, M., and Moller, T., 2005. "Evaluating visualizations: do expert reviews work?". Computer Graphics and Applications, IEEE, 25(5), pp. 8-11.

[45] Hart, S. G., and Staveland, L. E., 1988. "Development of nasa-tlx (task load index): Results of empirical and theoretical research". Advances in Psychology, 52, pp. 139-183.

[46] Brooke, J., 1996. "SUS-A quick and dirty usability scale”. Usability Evaluation in Industry, pp. 189-194.

[47] Shneiderman, B., 2001. "Inventing discovery tools: Combining information visualization with data mining". In Discovery Science, Springer, pp. 17-28. 